\title{
Exploring coronial determination of intent for poisoning-related deaths in Australia, 2001-2013
}

Kate Churruca* and Rebecca Mitchell

\begin{abstract}
Background: In countries like the United States and the United Kingdom, systematic variation in the classification of intent in pharmaceutical poisoning deaths have been identified between jurisdictions. This study aimed to explore whether the coronial determination of intent (unintentional, intentional, undetermined) for pharmaceutical-related poisoning deaths may have affected death rates over time and by jurisdiction in Australia.

Methods: A retrospective examination of mortality records in the National Coronial Information System (NCIS) during 1 January 2001 to 31 December 2013 was conducted. The NCIS is a national internet-based data storage and retrieval system for deaths that were notified to a coroner. Pharmaceutical deaths due to unintentional, intentional or undetermined intent were identified using the NCIS classification. Proportions of the different intent classifications and the mortality rates by intent over time were compared between jurisdictions.

Results: There were 17,895 pharmaceutical-related poisoning deaths in Australia between 2001 and 2013 that had closed cases in the NCIS. Proportions of deaths classified as unintentional (48.3-66.3\%), intentional (24.7-35.9\%) and undetermined (6.7-24.7\%) varied significantly among Australian jurisdictions. There were significant increases in the rate of classification of unintentional poisoning for some states, and significant increases in intentional poisoning classification in Western Australia, and decreases in New South Wales and Victoria. There was no significant change in classification of undetermined intent.

Conclusions: Significant variation in classifications of intent, both between state jurisdictions and over time, may be the result of regional differences in demographics and increases in prescription drug misuse. However, the inconsistent use of 'undetermined' intent between state jurisdictions suggests coroners may experience varying difficulty in retrospectively ruling on intent in the equivocal circumstances of pharmaceutical poisoning. The widespread use of psychological autopsy may assist coroners to classify intent, while the implementation of new classifications for pharmaceutical poisoning death may overcome some of the inherent difficulty in intent classification and improve the potential for injury surveillance irrespective of intent.
\end{abstract}

Keywords: Coroners systems, Suicide, Drug and alcohol policy, Medico-legal databases, Coding and classification

\footnotetext{
* Correspondence: kate.churruca@mq.edu.au

Australian Institute of Health Innovation, Macquarie University, Level 6, 75

Talavera Road, North Ryde, Sydney, NSW 2109, Australia
} 


\section{Background}

Intentional self-harm and suicide represent a significant public health problem worldwide [1]. In Australia, death due to intentional self-harm occurs at a rate of 10.1 per 100,000 population [2], which is comparable with the United States (US), New Zealand and Canada, but higher than the United Kingdom (UK) and a number of other European and Asian nations [3]. Indeed, such figures likely under enumerate the total number of self-harm deaths, because it is often difficult for coroners and medical examiners, who investigate injury deaths, to make an unequivocal decision of the individual's intent, such as whether the death may have been intentional or unintentional if the person has not left a note, or there is no documented history of mental illness $[4,5]$. In addition to the availability of information affecting determination of intent, a coroner's suspicions of suicide may be outweighed by social pressures, such as the stigma of suicide, moral or religious concerns, and, in some cases (e.g., suicide within 13 months of policy commencement), the loss of life insurance benefits $[6,7]$. These issues likely lead some instances of intentional self-harm deaths to be misclassified as 'undetermined' or 'unintentional' [7].

Deaths involving pharmaceuticals can be especially difficult for coroners to determine intent [8], because while death may not have necessarily been the intended outcome in circumstances of drug overdose, the ingestion of an intoxicating, often harm-causing, substance was also not unintentional [9]. Furthermore, use of some medicinal drugs is understood to inhibit an individual's mental capacity to form an intent with regard to death [7]. Therefore, while substance use is a risk factor for suicide, the involvement of drugs in a death may reduce the likelihood that the death is classified as suicide [10]. Any instance of coroner misclassification of intent not only affects the estimation of suicide numbers, but also unintentional poisoning, making it difficult to assess each of their burdens. Misclassification of unintentional and intentional poisoning may impact upon the ability to understand risk factors for both, and gain support for, and adequately tailor, prevention initiatives [7], which have traditionally focused on different factors: environment for unintentional injuries and behaviour for intentional injury [11].

In the US, the frequency of classification of deaths due to 'undetermined' intent has been found to vary considerably by state (from $<4 \%$ to $85 \%$ of poisoning deaths), as has the level of documentation of specific drugs on the death certificate, fluctuations that may be due to differing resources, investigatory systems (e.g., centralized medical examiner versus county coroner), methods of documenting, and context [12]. Breiding and Wiersema [13] also found that states with the highest rates of undetermined intent ranked behind most others on mean mortality rates of both intentional and unintentional pharmaceutical poisoning deaths. Furthermore, an association has been established between the decline in the suicide rate and the large increase in unintentional poisonings from 1987 to 2006, which suggests some potential suicides are being classified as unintentional deaths [14]. Comparable to these trends, in the UK, McLean [15] found that jurisdictions with the lowest rates of suicide had the highest rates of 'narrative' verdicts, which involves the factual reporting of circumstance for a death that does not simply fit standard verdicts (e.g., natural causes, suicide); on the other hand, jurisdictions with the lowest rates of narrative verdicts had the highest rates of suicide.

In Australia, while deaths due to unintentional pharmaceutical poisoning were on the decline to 2002, from 6.8 deaths per 100,000 population to 3.4 deaths, they have since been increasing (4.5 deaths per 100,000 in 2009-10), corresponding with increases in the use and misuse of prescription opioids [16, 17]. However, little is known about whether there are differences in the classification of intent in Australia for pharmaceutical poisoning, such as less frequent classification of intentional poisoning or more frequent use of undetermined intent for pharmaceutical poisoning. As with countries like the US and UK, there is a lack of standardisation of the procedures for determining intent, with Australian jurisdictions (i.e., state or territory coronial office) having different legislation and coroners not typically legally required to determine intent. Coders for the National Coronial Information System (NCIS), who categorize cases of coroner reported death nationally, have previously reported difficulties in coding intent, particularly for overdose deaths, and coders vary in the documentation and procedures they rely on for this classification [18]. This suggest the likelihood of variability between jurisdictions [19] impacting on injury surveillance of pharmaceutical poisoning by intent. This study aims to explore whether the coronial determination of intent for pharmaceutical-related poisoning deaths may have affected mortality incidence rates for different intent classifications over time and by jurisdiction in Australia.

\section{Method}

A retrospective examination of mortality records in the NCIS during 1 January 2001 to 31 December 2013 was conducted. Ethical approval was obtained from the Macquarie University Human Research Ethics Committee (reference no: 5,201,500,660), the Victorian State Government Justice Human Research Ethics Committee (CF/15/ 16426) and the Western Australia Coronial Ethics Committee (EC16/2015).

\section{Data collection}

The NCIS is a national internet-based data storage and retrieval system for deaths that were notified to a coroner. 
All deaths not presumed to have a natural cause (including injury-related deaths) should be notified to a coroner in Australia. The NCIS contains information on every death reported to an Australian coroner since July 2000 (January 2001 for Queensland). Within the NCIS, detailed information regarding the circumstances of the death is available on 'closed cases' (i.e., coronial cases finalized by a coroner) and minimal information is available on 'open cases' (excluding Western Australia, where information is only available on 'closed cases').

The NCIS includes information on the cause and circumstances of the death (e.g., mechanism of injury, intent), and demographic information of the person who died, with many the death records having reports (autopsy, toxicology, police) and coronial findings attached. Pharmaceutical-related poisoning was identified using the NCIS classifications for mechanism of injury of poisoning by solid substance (NCIS: 6.01.1) OR poisoning by liquid substance (NCIS: 6.01.2) AND object or substance producing injury is pharmaceutical substance for human use (NCIS: 20, excluding alcohol: 20.40) [20]. A search of poisoning by multiple substances (NCIS: 6.01.4) was also conducted; any cases containing a pharmaceutical in their object or substance producing injury were included. Intent was identified using the NCIS intent at case completion of unintentional (NCIS: 1), intentional self-harm (NCIS: 2), or undetermined intent (NCIS: 7). Any cases classified with intent as unlikely to be known (NCIS: 999), or with intent left blank, were also coded as undetermined intent [5]. All other possible intents (NCIS: 3-6) were classified as 'other'.

\section{Data management and analysis}

All analyses were performed using SAS version 9.4 [21]. Descriptive statistics were conducted. Chi-square analysis was conducted to examine the differences in the proportion of intent classifications by jurisdiction for the whole 13-year period. Crude mortality incidence rates over time were calculated using denominator data obtained from the ABS population estimates [22] for the whole of Australia, and for the individual states of New South Wales (NSW), Victoria (VIC), Queensland (QLD), South Australia (SA), and Western Australia (WA). Tasmania (TAS), the Northern Territory (NT) and the Australian Capital Territory (ACT) were excluded from rates due to low cell frequency. Due to over-dispersion, negative binomial regression analyses using the number of deaths as the dependent variable and year of death and age group as independent variables with an offset of the log of the population were used to examine the statistical significance of changes in the trend over time in mortality incidence rates by intent for the whole of Australia and then within each state [23]. Age-standardised mortality rates were not able to be calculated due to $<5$ cell size by age group and gender for some jurisdictions.

\section{Results}

There were 17,895 deaths as a result of pharmaceutical poisoning in Australia from 2001 to 2013 identified using the NCIS. The number of deaths due to pharmaceutical poisoning increased over this 13-year period, peaking in 2012 with 1712 deaths. Close to two-thirds of the sample were male (64.3\%). The highest proportion of deaths occurred in those in the middle adulthood years of 30-49 years (50.4\%). Unintentional poisoning was the most commonly classified intent, with 9951 deaths (55.6\%), and NSW had the highest number of pharmaceutical poisoning deaths (Table 1).

Examination of the frequency of intent classification by state suggested some variability. The proportion of deaths that were classified as unintentional (48.3-66.3\%), intentional (24.7-35.9\%) and undetermined (6.7-24.7\%) varied significantly for the eight Australian jurisdictions during the 13-year study period of study (Table 2). Crude mortality rates for unintentional, intentional, and undetermined intent further indicated variability in the classification of intent over time and between the different states of Australia (Fig. 1). Most of this variation was in the classification of unintentional poisoning. This variation in unintentional poisoning was evident for all Australia, as well as between Australian states (e.g., variation between WA and VIC) and within most states over time, with NSW the only exception to this. Results of the individual negative binomial regression analyses showed significant increases in the rate of classification of unintentional poisoning for the model with the whole of Australia, and then models for a number of states (QLD, SA, WA). The rate of intentional pharmaceutical poisoning also significantly increased for the model of the whole of Australia and for that of WA; on the other hand, classification of intentional poisoning significantly decreased in NSW and VIC. While changes in the rates of undetermined intent varied between the models for individual states, with some rates increasing (NSW, VIC, WA) and others decreasing (QLD, SA), none of these changes were significant (Table 3).

\section{Discussion}

This study explored whether the coronial determination of intent for pharmaceutical-related poisoning deaths may have affected mortality incidence rates over time and by state in Australia using the NCIS data collection from 2001 to 2013. There was a rise in the number of pharmaceutical poisoning deaths over the 13-year period of study, with an examination of the annual percent change in mortality rates for both unintentional and 
Table 1 Characteristics of individuals who died as a result of pharmaceutical poisoning, Australia, 2001-2013

\begin{tabular}{|c|c|c|c|c|c|c|}
\hline & \multicolumn{2}{|c|}{$\begin{array}{l}\text { All persons } \\
(17,895)\end{array}$} & \multicolumn{2}{|c|}{$\begin{array}{l}\text { Male } \\
(11,509)\end{array}$} & \multicolumn{2}{|c|}{$\begin{array}{l}\text { Female } \\
\text { (6386) }\end{array}$} \\
\hline & $n$ & $\%$ & $n$ & $\%$ & $\mathrm{n}$ & $\%$ \\
\hline \multicolumn{7}{|l|}{ Year of death } \\
\hline 2001 & 1085 & 6.1 & 678 & 5.9 & 407 & 6.4 \\
\hline 2002 & 1086 & 6.1 & 700 & 6.1 & 386 & 6.0 \\
\hline 2003 & 1169 & 6.5 & 750 & 6.5 & 419 & 6.6 \\
\hline 2004 & 1254 & 7.0 & 837 & 7.3 & 417 & 6.5 \\
\hline 2005 & 1355 & 7.6 & 881 & 7.7 & 474 & 7.4 \\
\hline 2006 & 1196 & 6.8 & 755 & 6.6 & 441 & 6.9 \\
\hline 2007 & 1435 & 8.0 & 871 & 7.6 & 564 & 8.8 \\
\hline 2008 & 1520 & 8.5 & 1002 & 8.7 & 518 & 8.1 \\
\hline 2009 & 1602 & 9.0 & 1048 & 9.1 & 554 & 8.7 \\
\hline 2010 & 1465 & 8.2 & 945 & 8.2 & 520 & 8.1 \\
\hline 2011 & 1549 & 8.7 & 1022 & 8.9 & 527 & 8.3 \\
\hline 2012 & 1712 & 9.6 & 1099 & 9.6 & 613 & 9.6 \\
\hline 2013 & 1467 & 8.2 & 921 & 8.0 & 546 & 8.6 \\
\hline \multicolumn{7}{|l|}{ Age groups $^{a}$} \\
\hline 0-19 & 453 & 2.5 & 300 & 2.6 & 153 & 2.4 \\
\hline $20-29$ & 3227 & 18.0 & 2398 & 20.8 & 829 & 13.0 \\
\hline $30-39$ & 4766 & 26.6 & 3409 & 29.6 & 1357 & 21.3 \\
\hline $40-49$ & 4267 & 23.8 & 2771 & 24.1 & 1496 & 23.4 \\
\hline $50-59$ & 2861 & 16.0 & 1559 & 13.6 & 1302 & 20.4 \\
\hline $60-69$ & 1230 & 6.9 & 593 & 5.2 & 637 & 10.0 \\
\hline $70+$ & 1088 & 6.1 & 476 & 4.1 & 612 & 9.6 \\
\hline \multicolumn{7}{|l|}{ Intent } \\
\hline Unintentional & 9951 & 55.6 & 6974 & 60.6 & 2977 & 46.6 \\
\hline Intentional & 5331 & 29.8 & 2966 & 25.8 & 2365 & 37.0 \\
\hline Undetermined & 2534 & 14.2 & 1534 & 13.3 & 1000 & 15.7 \\
\hline Other $^{\mathrm{b}}$ & 79 & 0.4 & 35 & 0.3 & 44 & 0.7 \\
\hline \multicolumn{7}{|l|}{ State } \\
\hline NSW & 5264 & 29.4 & 3404 & 29.6 & 1860 & 29.1 \\
\hline $\mathrm{VIC}$ & 4411 & 24.7 & 2854 & 24.8 & 1557 & 24.4 \\
\hline QLD & 3289 & 18.9 & 2080 & 18.1 & 1209 & 18.9 \\
\hline SA & 1374 & 7.7 & 830 & 7.2 & 544 & 8.5 \\
\hline WA & 2486 & 13.9 & 1685 & 14.6 & 801 & 12.5 \\
\hline TAS & 586 & 3.3 & 341 & 3.0 & 245 & 3.8 \\
\hline NT & 216 & 1.2 & 161 & 1.4 & 55 & 0.9 \\
\hline ACT & 269 & 1.5 & 154 & 1.3 & 115 & 1.8 \\
\hline
\end{tabular}

${ }^{a}$ Three male cases are not reported because they were missing age details ${ }^{b}$ Other intent includes Legal Intervention, Operations of War, Civil Conflict and Acts of Terrorism, Other Specified Intent [20]

intentional poisoning also showing significant increases over time, except for intentional poisoning in NSW and VIC where there were significant decreases. Overall, the number of pharmaceutical deaths identified in this study
Table 2 Frequency and percentage of deaths through pharmaceutical poisoning by intent for all Australia and each state or territory, 2001-2013

\begin{tabular}{|c|c|c|c|c|c|c|c|}
\hline \multirow[t]{3}{*}{ Location } & \multicolumn{6}{|c|}{ Intent Classification ${ }^{a}$} & \multirow[t]{3}{*}{$x^{2}(d f)$} \\
\hline & \multicolumn{2}{|c|}{ Unintentional } & \multicolumn{2}{|c|}{ Intentional } & \multicolumn{2}{|c|}{ Undetermined } & \\
\hline & $n$ & $\%$ & $n$ & $\%$ & $n$ & $\%$ & \\
\hline All Australia & 9951 & 55.9 & 5331 & 29.9 & 2534 & 14.2 & \\
\hline \multicolumn{8}{|l|}{ States } \\
\hline NSW & 3020 & 57.6 & 1529 & 29.2 & 693 & 13.2 & \multirow[t]{8}{*}{$404.60(14)^{* * * *}$} \\
\hline VIC & 2473 & 56.4 & 1116 & 25.5 & 793 & 18.1 & \\
\hline QLD & 1718 & 52.4 & 1178 & 35.9 & 386 & 11.8 & \\
\hline SA & 664 & 48.5 & 366 & 26.8 & 338 & 24.7 & \\
\hline WA & 1479 & 59.7 & 835 & 33.7 & 165 & 6.7 & \\
\hline TAS & 299 & 51.6 & 179 & 30.7 & 102 & 17.6 & \\
\hline NT & 121 & 56.0 & 62 & 28.7 & 33 & 15.3 & \\
\hline $\mathrm{ACT}$ & 177 & 66.3 & 66 & 24.7 & 24 & 9.0 & \\
\hline
\end{tabular}

$* * * * p<0.0001$

a'Other' intent not reported

were similar to those found by Henley and Harrison [17], who reported 1496 cases of pharmaceutical poisoning mortality for 2009-10 and comparable proportions of unintentional, intentional and undetermined intent deaths.

The classification of intent, when collapsed across the 13-year period of study, was found to vary significantly between Australian states, with much higher proportions of unintentional poisoning in the ACT, and undetermined intent in VIC, SA, and TAS. It is unclear at this stage to what extent some of this state-based variation in the intent classification for poisoning deaths reflects real difference in manner of death between different states versus inconsistent classification practices between jurisdictions. There is considerable variation in the population characteristics among Australian states and territories. For example, the ACT has higher number of females and younger people in their population, and approximately $34 \%$ are employed by the Commonwealth Government of Australia [24]. On the other hand, in WA and the NT, a larger proportion of the population is male, and, for WA in particular, employed in highpaying, 'fly-in, fly-out' mining work $[25,26]$. These regional differences have likely affected the type of drug and patterns of drug-taking [27], hence contributing to the different proportions of intent classifications between states (e.g., higher proportion of unintentional poisoning in $\mathrm{ACT}$, and intentional in WA). At the same time, the variation between some states in the proportion of undetermined intent classification (i.e., 6.7-24.7\%) seems to suggest more than just an increased frequency of ambiguous deaths within these jurisdictions. In this vein, a US study by Warner, et al. [12] noted that large differences 


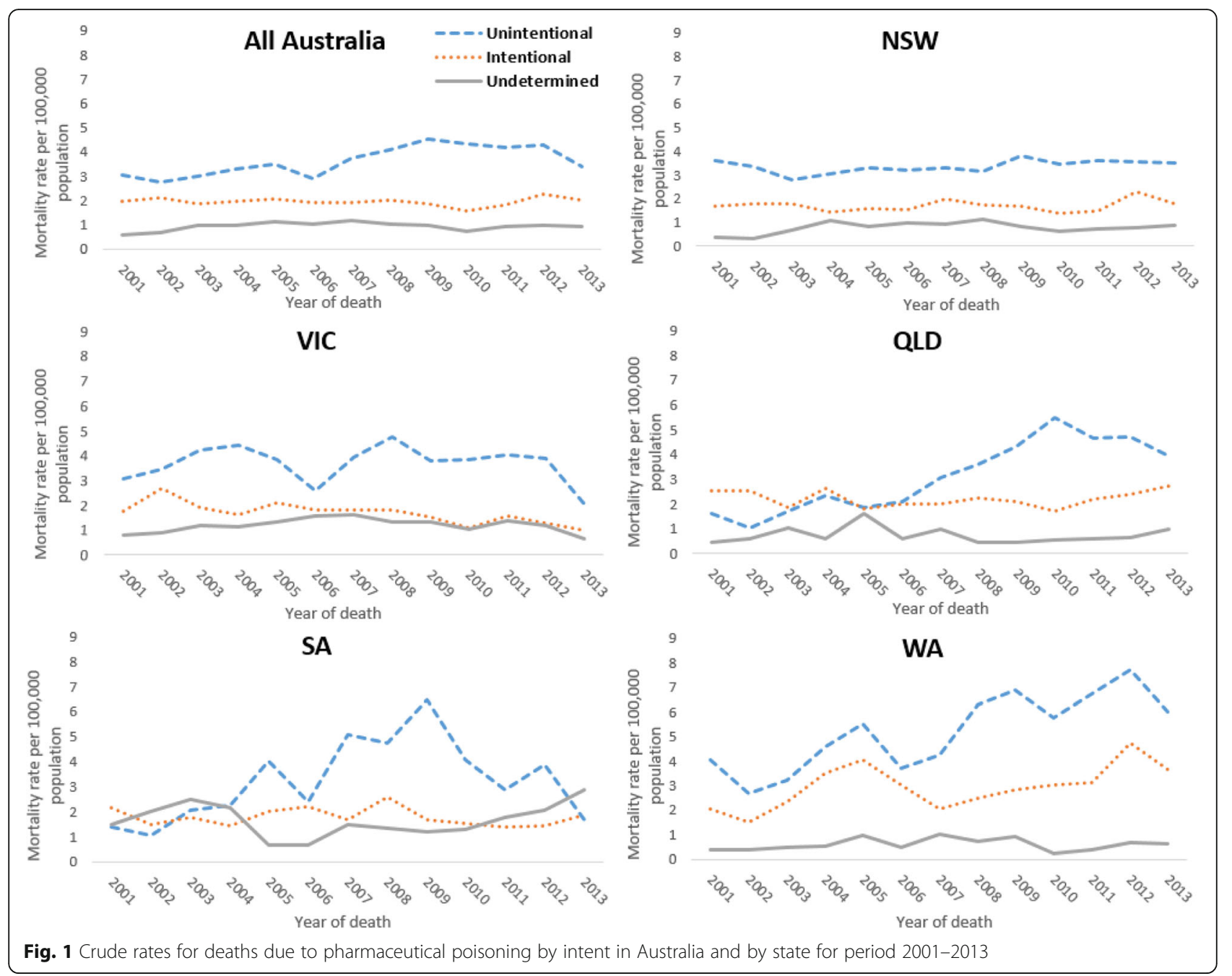

between states in the use of the undetermined intent classification were likely due to a lack of standardisation in the methods of investigating deaths and the criteria used to determine intent.

The present study also found changes in the classification of intent for pharmaceutical deaths for some states over time, such as increases in both intentional and unintentional poisoning in WA, and decreases in intentional poisoning in VIC and NSW. However, contrary to trends from the US reported by Breiding and Wiersema [13] and Rockett, et al. [14], significant declines in the rate of intentional poisoning, such as in NSW and VIC, did not correspond with increases in either rates of unintentional or undetermined intent. Indeed, changes in the rate of classification of undetermined intent for all states were nonsignificant, though graphically crude rates for SA and QLD did show the anticipated trend of a negative relationship between undetermined intent and intentional poisoning. Finally, for WA rates of both unintentional and intentional poisoning rose over time, but did not correspond to any significant changes in the rate of undetermined intent.

Overall, findings suggest mortality incidence rates may be affected by some variations in the classification of intent in pharmaceutical poisoning deaths between coronial jurisdictions in Australia, specifically regarding the frequency with which the classification of undetermined intent is utilized between some states. The evidence to suggest changes in classification of intent over time is more tentative, and does not follow the trend found in the US [14]; instead there were significant increases in both unintentional and intentional poisoning. The trends identified in this study may therefore reflect real changes in the incidence of pharmaceutical poisoning for the different intent types. For example, the increase in unintentional poisoning over time may reflect changes in manner of death between states due to differences in their sociodemographic characteristics, and particularly increases in the misuse of prescription pharmaceuticals, which are often classified under 'unintentional' [28]. Future 
Table 3 Regression of rates of change in intent classification for pharmaceutical poisoning deaths in Australia and by state, 2001-2013

\begin{tabular}{|c|c|c|}
\hline Location & Change & $95 \% \mathrm{Cl}$ \\
\hline \multicolumn{3}{|l|}{ All Australia } \\
\hline Unintentional & $3.47^{* * * *}$ & $2.49-4.46$ \\
\hline Intentional & $1.83^{* *}$ & $0.76-2.91$ \\
\hline Undetermined & 0.22 & $-0.96-1.41$ \\
\hline \multicolumn{3}{|l|}{ NSW } \\
\hline Unintentional & 0.99 & $-0.70-2.71$ \\
\hline Intentional & $-5.18^{* *}$ & $-8.72--1.50$ \\
\hline Undetermined & 1.43 & $-0.91-3.82$ \\
\hline \multicolumn{3}{|l|}{ VIC } \\
\hline Unintentional & -0.07 & $-1.99-1.88$ \\
\hline Intentional & $-5.05^{* * * *}$ & $-7.30--2.75$ \\
\hline Undetermined & 0.98 & $-1.02-3.03$ \\
\hline \multicolumn{3}{|l|}{ QLD } \\
\hline Unintentional & $10.26^{* * * *}$ & $7.91-12.65$ \\
\hline Intentional & 0.84 & $-1.32-3.05$ \\
\hline Undetermined & -0.85 & $-3.59-1.98$ \\
\hline \multicolumn{3}{|l|}{ SA } \\
\hline Unintentional & $3.95^{* * *}$ & $1.64-6.31$ \\
\hline Intentional & 1.37 & $-0.99-3.79$ \\
\hline Undetermined & -0.49 & $-3.21-2.32$ \\
\hline \multicolumn{3}{|l|}{ WA } \\
\hline Unintentional & $6.66^{* * * *}$ & $3.90-9.50$ \\
\hline Intentional & $4.40^{* * *}$ & $2.14-6.72$ \\
\hline Undetermined & 0.61 & $-3.60-4.99$ \\
\hline
\end{tabular}

research exploring differences in patterns of drug-taking and pharmaceutical poisoning between Australian states over time may provide further insights into the reasons for diverging trends among jurisdictions with regard to intent.

Nevertheless, the large variability in usage of the classification of 'undetermined intent' in this study between coronial jurisdictions suggests there may be a lack of consensus among coroners in Australian states about how to classify some deaths resulting from pharmaceutical poisoning. This may still limit the ability to accurately determine the extent of intentional and unintentional poisoning at a state and national level, and to then address them accordingly through the identification of risk factors and targeted interventions [6, 7]. The more widespread use of psychological autopsies might be beneficial in reducing the use of 'undetermined' intent at the time in which coroners make their determination, by providing them more information and expertise to make an accurate decision [4]. Psychological autopsy involves comprehensive psychological information about the deceased person being collected through extensive interviews with family members and other close intimate persons, as well as through medical, psychiatric and legal records; this information may be assessed by psychiatrists and other mental health professionals to accurately determine the role of intent in the death [29]. While not currently standard practice in nations like Australia [30], they have been found to improve the precision of classification of intent. Moreover, psychiatrists and suicide researchers have been found more likely to identify deaths that were previously classified by coroners as undetermined intent to be intentional [31]. However, the implementation of psychological autopsies would require the allocation of greater resources to coronial offices. As a starting point, national coronial practice guidelines and aids regarding the classification of intent [6], particularly for pharmaceutical poisonings, may be useful in improving consistency among the classifications between jurisdictions. The linkage of administrative health datasets may also provide an opportunity into the future to gather additional information to support the determination of intent, such as the deceased having a record of hospitalisation for self-harm, drug use, or mental illness.

As an alternative to standardisation of existing procedures, the implementation of new concepts to classify deaths due to pharmaceutical poisoning death may overcome some of the inherent difficulty in attempting to retrospectively determine the role of intent in equivocal circumstances. In this vein, Rockett, et al. [14] have proposed the addition of a category, death from drug selfintoxication (DDSI), which would apply across intent classifications to indicate deaths where pharmaceuticals were deliberately ingested for purposes of intoxication (e.g., overdose deaths). Similar to this, using examples of equivocal gunshot death, Obenson [32] suggested that the classification of suicide could be supplanted by that of 'self-inflicted' death, which would carry less stigma, reduce the subjectivity of medical examiners and pathologists ruling on intent, and defer this decision to other more qualified or better resourced facilities (e.g., departments conducting psychological autopsies).

Hence, the use of a DDSI style classification in Australia would allow for better tracking of the problematic use of pharmaceuticals and sidestep some of the difficulty for coroners in determining a construct like intent in pharmaceutical poisoning, where death might not be intended but intoxication is, and the potential for self-harm is recognized. This categorisation could also trigger resourcing for further investigation of DDSI deaths to determine intent, such as through psychological autopsy. Monitoring pharmaceutical usage has received increasing attention in Australia recently [33], with the rise of prescription drug misuse. The implementation of DDSI classification could work alongside other proposed strategies such as real- 
time prescription drug monitoring, which attempts to track and subsequently address problematic prescribing, dispensing and use of some prescription drugs to avoid unintentional poisoning [33].

These findings were strengthened by the populationbased nature of the study, the use of a large cohort and inclusion of data from a relatively long period of time, which thereby enabled greater capacity to identify trends. At the same time, there were some limitations to this study. First, due to the small numbers of deaths for some age groups by sex, age and sex standardized mortality rates were not able to be calculated for each intent classification, which may have affected comparisons between some states. The ICD-10 codes, which are used by the ABS to classify mortality data for official reporting, were also not used in this study to select cases of pharmaceutical poisoning nor to classify intent. This may lead to some discrepancies between the number and rate of poisoning deaths identified in the current study and other reported Australian national mortality figures using Cause of Death-Unit Record File mortality data, particularly as there is the possibility of incorrect classification of poisoning deaths in the NCIS. However, the ABS relies upon coronial investigations and NCIS data for their classification of cause of death and intent [5]. Moreover, the similarity in the number of poisoning deaths and proportion of different intent classifications in the current study to those identified by Henley and Harrison [17] suggests any such discrepancies were minor.

This study was confined to the examination of NCIS closed cases, which may have reduced the number of pharmaceutical poisoning-related deaths, especially for later years where coronial investigations of deaths may not yet have concluded [34]. This may have affected rates of intent classification over time and particularly differences between states. For example, while Studdert, et al. [35] found the average length of time for closure of coronial cases involving suicide or undetermined intent was relatively short, case closure was affected by jurisdiction; however, this was only for the smaller subset of cases requiring a full inquest. It is also possible that the numbers of deaths by intent reported in this study reflect not only cases of intent as classified by coroners, but NCIS coders too, with De Leo, et al. [6] finding that in $29 \%$ of the 988 NCIS cases they reviewed, the coroner made no reference to intent. However, this issue is reportedly more likely to occur for deaths with less equivocal circumstances (e.g. hanging, motor vehicle exhaust) than pharmaceutical poisoning deaths. Finally, in this study $5.0 \%$ of deaths had no classification for their intent at case completion. It is unclear whether this was due to oversight, reluctance among some NCIS coders to classify intent, or some other reason, and following the ABS guidelines, these blank cases were treated as undetermined intent [5].
However, it is worth noting that the proportion of deaths that did not have a classified intent at case completion varied by jurisdiction (2.3-11.1\%), which may have affected rates of undetermined intent by state.

Finally, research suggests that the way in which intent is classified over time is associated with the type of drugs ingested (e.g., prescription or recreational) [13]. Any associations between intent classification and the types of pharmaceuticals involved in the death were unable to be examined for the present study, however, because $35.8 \%$ of the sample had their primary pharmaceutical object or substance producing injury classified as other specified multiple substances (NCIS: 20.50.98), which precluded meaningful analysis of any relationship between specific drugs and intent classification. This is also unfortunate because the ability to identify associations between specific and multiple pharmaceuticals and intent may have significant implications for prevention of injury and death [12]. This issue resonates with previous research showing that even on coroner reports and findings, drugs and specific drugs are often underreported as a cause of death and, hence, underacknowledged as an opportunity for future prevention $[12,36]$. However, usage of multiple drugs is common practice, particularly among older people in Australia [37], with around twothirds of individuals aged 60 years or older using four or more medications [38]. As such, revising coding practices for pharmaceutical deaths documented in medico-legal databases, such as the NCIS, to allow for the coding of individual drugs in circumstances were multiple drugs are involved in the death is worthwhile pursuit to enable further research on the effect of drug type on intent classification, in addition to future injury surveillance and prevention. Alternatively, the use of Cause of Death-Unit Record File data, which involves classification by the ABS using ICD-10 codes, would enable future research to explore whether drug-type affects the way coroners determine intent.

\section{Conclusion}

This study found that there were significant differences between jurisdictions in the proportion of pharmaceuticalrelated poisoning deaths classified as intentional, unintentional and undetermined intent during 2001-2013 in Australia. Moreover, there were significant changes in the classification of intent for some states over time, with decreases in intentional poisoning (VIC, NSW), and, particularly, increases in unintentional poisoning overall in Australia and some states (QLD). The variability between jurisdictions in the use of the classification of 'undetermined intent' suggests there may be a need for greater standardisation of procedures for classifying deaths due to pharmaceutical poisoning in Australia. 


\section{Abbreviations}

ABS: Australian Bureau of Statistics; ACT: Australian Capital Territory; ICD10: International Statistical Classification of Diseases and Related Health Problems version 10; NCIS: National Coronial Information System; NSW: New South Wales; NT: Northern Territory; QLD: Queensland; SA: South Australia; TAS: Tasmania; VIC: Victoria

\section{Acknowledgements}

The authors wish to thank the National Coronial Information System for providing the data and for assistance with extraction.

\section{Funding}

The authors declare no funding.

\section{Availability of data and materials}

The data that support the findings of this study are available from the National Coronial Information System but restrictions apply to the availability of these data, which were used under license for the current study, and so are not publicly available. Data are however available from the authors upon reasonable request and with permission of the data custodian and the ethics committees. A license to access and use the National Coronial Information System is also required.

\section{Authors' contributions}

RM developed the study concept and design. KC acquired the data, conducted the analysis and drafted the manuscript. KC and RM were involved in interpretation and critical revision of the manuscript. All authors read and approved the final manuscript.

\section{Ethics approval and consent to participate}

Ethical approval was obtained from the Macquarie University Human Research Ethics Committee (reference no: 5,201,500,660), the Victorian State Government Justice Human Research Ethics Committee (CF/15/16426) and the Western Australia Coronial Ethics Committee (EC16/2015). A waiver of consent was granted by the ethics committees.

\section{Consent for publication}

Not applicable.

\section{Competing interests}

The authors declare that they have no competing interests.

\section{Publisher's Note}

Springer Nature remains neutral with regard to jurisdictional claims in published maps and institutional affiliations.

Received: 9 February 2017 Accepted: 26 July 2017

Published online: 01 August 2017

\section{References}

1. Bertolote JM, Fleischmann A. A global perspective in the epidemiology of suicide. Suicidologi. 2002;7:6-8. doi:10.5617/suicidologi.2330.

2. AlHW, Harrison JE, Henley G. Suicide and hospitalised self-harm in Australia: trends and analysis. In: Injury research and statistics series no. 93. Cat. no. INJCAT 169. Canberra: AlHW; 2014.

3. World Health Organization: SDG target 3.4, indicator 3.4 .2 suicide mortality rate. In: Sustainable development goals. Geneva, Switzerland: WHO; 2012.

4. Berman $A L$, Josselson AR, Jobes DA. The impact of psychological autopsies on medical examiners' determination of manner of death. J Forensic Sci. 1986;31:177-89.

5. ABS. Explanatory notes. Canberra: ABS; 2016.

6. De Leo D, Dudley MJ, Aebersold CJ, Mendoza JA, Barnes MA, Harrison JE, Ranson DL. Achieving standardised reporting of suicide in Australia: rationale and program for change. Med J Aust. 2010;192:452-6.

7. Rosenberg M, Davidson L, Smith J, Berman A, Buzbee H, Gantner G, Gay G, Moore-Lewis B, Mills D, Murray D, et al. Operational criteria for the determination of suicide. J Forensic Sci. 1988;33:1445-56.

8. Lindqvist $P$, Gustafsson L. Suicide classification-clues and their use: a study of 122 cases of suicide and undetermined manner of death. Forensic Sci Int 2002;128:136-40. http://dx.doi.org/10.1016/S0379-0738(02)00188-3
9. Rockett IR, Smith GS, Caine ED, Kapusta ND, Hanzlick RL, Larkin GL, Naylor CP, Nolte KB, Miller TR, Putnam SL. Confronting death from drug self-intoxication (DDSI): prevention through a better definition. Am J Public Health. 2014;104:e49-55.

10. Hempstead K: Manner of death and circumstances in fatal poisonings: evidence from New Jersey. Inj Prev 2006;12:ii44-ii48. 10.1136/ip.2006.012583.

11. Cohen L, Miller T, Sheppard MA, Gordon E, Gantz T, Atnafou R. Bridging the gap: bringing together intentional and unintentional injury prevention efforts to improve health and well being. J Saf Res. 2003;34:473-83. http:// dx.doi.org/10.1016/j.jsr.2003.03.005

12. Warner M, Paulozzi L, Nolte K, Davis GG, Nelson L. State variation in certifying manner of death and drugs involved in drug intoxication deaths. Acad Forensic Pathol. 2013;3:231-7.

13. Breiding MJ, Wiersema B: Variability of undetermined manner of death classification in the US. Inj Prev 2006;12:ii49-ii54. 10.1136/ip.2006.012591.

14. Rockett IR, Hobbs G, De Leo D, Stack S, Frost JL, Ducatman AM, Kapusta ND, Walker RL: Suicide and unintentional poisoning mortality trends in the United States, 1987-2006: two unrelated phenomena? BMC Public Health 2010;10 journal article:1-11. 10.1186/1471-2458-10-705.

15. McLean M. Coroner consistency - the 10-jurisdiction, 10-year, postcode lottery? Med Sci Law. 2014;55:102-12. doi:10.1177/0025802414526711.

16. Blanch B, Pearson S-A, Haber PS. An overview of the patterns of prescription opioid use, costs and related harms in Australia. Br J Clin Pharmacol. 2014 78:1159-66. doi:10.1111/bcp.12446.

17. AlHW, Henley G, Harrison JE. Trends in injury deaths, Australia: 1999-00 to 2009-10. Canberra: AlHW; 2015.

18. Dodds L, Robinson KM, Daking L, Paul L. The concept of 'intent' within Australian coronial data: factors affecting the national coronial information system's classification of mortality attributable to intentional self-harm [online]. Health Inform Manag J. 2014;43:13-22.

19. Senate Community Affairs Reference Committee. Commonwealth response to the hidden toll: suicide in Australia. Canberra: Commonwealth of Australia; 2010

20. National Coroners Information System. Data dictionary for the National Coroners Information System. Version 2. Southbank: National Coroners Information System; 2007.

21. SAS Institute. SAS: statistical software, version 9.4. Cary, North Carolina: SAS Institute; 2014

22. Australian Bureau of Statistics: Australian demographic statistics. In: Cat. no 3101.0. Canberra: ABS; 2015.

23. Armitage $P$, Berry G, Matthews J. Statistical methods in medical research, fourth edition edn. Cornwell: Blackwell Science; 2002.

24. ABS. Centenary of Canberra. In: 4102.0 - Australian social trends. Canberra: ABS; 2013.

25. ABS. People. Canberra: ABS; 2014

26. ABS. Main features 2010. Canberra: ABS; 2011

27. FH MK. Fly-in fly-out: the challenges of transient populations in rural landscapes. In: Luck GW, Black R, Race D, editors. Demographic change in Australia's rural landscape. Edn. Netherlands: Springer; 2010. p. 353-74

28. Pilgrim JL, Yafistham SP, Gaya S, Saar E, Drummer OH: An update on oxycodone: lessons for death investigators in Australia. Forensic Sci Med Pathol 2015;11journal article:3-12. 10.1007/s12024-014-9624-x.

29. Isometsä ET. Psychological autopsy studies - a review. European Psychiatry. 2001;16:379-85. https://doi.org/10.1016/S0924-9338(01)00594-6.

30. National Committee for Standardised Reporting on Suicide: Submission community affairs committee inquiry into suicide in Australia. In. Sydney, NSW, AUS Suicide Prevention Australia; 2010.

31. Gunnell D, Bennewith O, Simkin S, Cooper J, Klineberg E, Rodway C, Sutton L, Steeg S, Wells C, Hawton K, et al. Time trends in coroners' use of different verdicts for possible suicides and their impact on officially reported incidence of suicide in England: 1990-2005. Psychol Med. 2013;43:1415-22. doi:10.1017/S0033291712002401

32. Obenson K. Suicide versus self-inflicted: is it time to change manner of death classification? Forensic Sci Med Pathol. 2013:9:576-7. doi:10.1007/ s12024-013-9478-7.

33. Ogeil RP, Heilbronn C, Lloyd B, Lubman DI. Prescription drug monitoring in Australia: capacity and coverage issues. Med J Aust. 2016;204:148. doi:10. 5694/mja15.00929.

34. Monthly Case Closure Statistics Summary [http://www.ncis.org.au/wpcontent/uploads/2011/12/Monthly-Case-Closure-Statistics-July-19-2016.pdf].

35. Studdert DM, Walter SJ, Kemp C, Sutherland G: Duration of death investigations that proceed to inquest in Australia. Inj Prev 2016. 10.1136/ injuryprev-2015-041933. 
36. Pilgrim JL, Gerostamoulos D, Drummer $\mathrm{OH}$. The role of toxicology interpretations in prevention of sudden death. Forensic Sci Med Pathol. 2012;8:263-9. doi:10.1007/s12024-011-9309-7.

37. Morgan T, Williamson M, Pirotta M, Stewart K, Myers S, Barnes J. A national census of medicines use: a 24-hour snapshot of Australians aged 50 years and older. Med J Aust. 2012;196

38. Elliot R. Problems with medication use in the elderly: an Australian perspective. J Pharm Pract Res. 2006;36:58-66.

Submit your next manuscript to BioMed Central and we will help you at every step:

- We accept pre-submission inquiries

- Our selector tool helps you to find the most relevant journal

- We provide round the clock customer support

- Convenient online submission

- Thorough peer review

- Inclusion in PubMed and all major indexing services

- Maximum visibility for your research

Submit your manuscript at www.biomedcentral.com/submit 